\title{
Global health response to crises needs reform
}

$\mathrm{C}$ alling the delays in declaring Ebola an international health emergency "significant and unjustifiable," an independent panel reviewing the World Health Organization's (WHO) handling of the crisis slammed the organization, its member countries and the wider United Nations (UN) in its report released July 7.

The report vindicates criticism from Médecins Sans Frontières (MSF), one of the main medical and humanitarian agencies on the ground during the epidemic, but MSF International President, Montréaler Dr. Joanne Liu said, "I don't take any pride in that." She says MSF's goal is "that next time around we can respond in a timely fashion when the red flags are raised."

The report detailed a perfect storm of factors causing delays and ineffective response during the epidemic's emergence in the spring of 2014, many involving the WHO and other UN agencies. In the years before the epidemic, WHO's member states held contributions steady, leading to a serious loss of purchasing power; budgets were tightened. The staff at the WHO's regional office for Africa was severely cut between 2011 and 2013 to only 10. The WHO failed to heed warnings from those staff and from groups such as MSF.

Smarting from criticism that it had been alarmist in declaring $\mathrm{H} 1 \mathrm{~N} 1$ influenza an international emergency, the WHO did not declare an emergency for Ebola until August 2014, although the scale of the epidemic had become clear in May or June. It also failed to challenge local governments for their inaction and lacked a culture to deal with large-scale, long-term and multicountry emergency response, said the report.

The report also documented a discon- nect between humanitarian and health agencies, both UN and governmental, as well as nongovernmental agencies. "The humanitarians did not quite understand the risk that Ebola brought," said panel chair Dame Barbara Stocking, former chief executive of Oxfam GB (Great Britain). The UN's World Food Programme is the lead on logistics and UNICEF leads on community engagement in emergencies, but she said both are unaccustomed to health emergencies and were not brought in quickly enough. A UN mission to deal with the crisis "took months" to set up. "What you don't need is a new UN mission being established right in the middle of a crisis."

Recommendations in the report aim at a major shake-up of the WHO, other UN agencies and the International Health Regulations. "We looked at WHO and its function as a health-emergency agency, and for it to function properly for that, it will need to be changed quite a lot. But we think that is perfectly possible," said Stocking.

One of the key recommendations is to have an "intermediate level" alert for disease outbreaks that are not yet an emergency. "The full public health emergency of international concern is very much a yes or no thing," Stocking said. "It is quite a difficult decision to take. Just waiting for the full final [decision] is too late. You're too far in by then. You need to do something that gets the world moving much earlier."

MSF pushed for such an intermediate alert. "If we don't somehow trigger a response before we hit the public health emergency of international concern, we're always going to wait for the catastrophe to happen," said Liu.

MSF is also pleased that the panel recommended incentives for countries to report outbreaks and disincentives or sanctions against countries such as Canada during the Ebola crisis - that bring in trade or travel bans in violation of the International Health Regulations. Such bans "not only frighten people out of declaring, but they also make it very difficult to deliver a proper response," as supplies and health care personnel may be unable to enter or leave affected countries, explained Stocking.

"The fear factor was hampering the response," agreed Liu. "The way it is right now, if you declare you have cholera in your country, you become the pariah of your region," with potentially disastrous economic consequences. "There needs to be a way to support these countries."

But Liu emphasized attitudinal shift. "Another thing that we cannot overlook is the political will to find an answer. Even if all this was fixed, if there is no political will in countries, we're still going to be faced with the same story."

Stocking says the report will feed into the current review of the International Health Regulations.

Liu said efforts to improve handling of future outbreaks are "great to a certain extent, but we should keep our eye on the ball of the current epidemic. We don't want to sound alarmist, but we need to be realistic. It is not over." Carolyn Brown, Ottawa, Ont.

CMAJ 2015. DOI:10.1503/cmaj.109-5115 